\title{
Purification and Characterization of Rat Liver Cytosol Catalase
}

\author{
Takehisa Sando ${ }^{1}$, Kunio Konno ${ }^{1}$, Nobuko Takei ${ }^{2}$, \\ Terufumi Sakamoto $^{3}$ and Tokuhiko Higashi ${ }^{4}$ \\ ${ }^{1}$ Department of Biochemistry, School of Medicine, Showa University, Shinagawa- \\ ku, Tokyo 142, ${ }^{2}$ Showa Medical Laboratory Technical School, Minamiazabu, \\ Minato-ku, Tokyo 106, ${ }^{3}$ Department of Physiological Chemistry, School of \\ Pharmaceutical Sciences, Showa University and ${ }^{4}$ Institute of Basic Medical \\ Sciences, University of Tsukuba, Ibaraki 305, Japan
}

\begin{abstract}
Rat liver cytosol catalase was purified by making use of its interaction with deoxycholate and by the various methods used to purify peroxisomal catalase. The purified preparation was both enzymatically and immunologically identical with the purified peroxisomal catalase, but differed in its chromatographic behaviour and electrophoretic mobility. Amino acid analysis also revealed a slight difference between the two catalase molecules.
\end{abstract}

When rat liver homogenates in $0.25 \mathrm{M}$ sucrose are fractionated subcellularly by conventional differential centrifugation, the supernatant (cytosol) fraction contains more than $40 \%$ of the total liver catalase (1.2). There has been discussion but no conclusion as to whether this catalase actually exists in the cytosol of liver cells or originates from peroxisomes destroyed by manipulation. The solution of this problem is important for investigations of the intracellular pathway of this enzyme protein after its synthesis on polysomes and for determining the biogenesis of peroxisomes.

Neither an enzymological nor an immunological difference has been found between the peroxisomal and supernatant catalase $(2,3)$. Higashi and his collaborators, however, have reported that the two catalases differ both in chromatographic (3) and in electrophoretic (4) behaviour. The evidence indicates that the supernatant catalase is less acidic than the peroxisomal catalase. Purification of the peroxisomal enzyme has been performed $(2,5)$, but so far purification of the supernatant catalase has not been successful.

We have found that the supernatant catalase interacts with deoxycholate (DOC), both being precipitable on adjusting the $\mathrm{pH}$ to 6 . By making use of this phenomenon in combination with the various procedures used to purify peroxisomal catalase $(2,5)$, rat liver supernatant catalase has been extensively purified and shows specific enzyme activity as high as that of the purified peroxisomal catalase. We have described purification steps for the supernatant (cytosol) catalase and report on its heterogeneous nature.

Abbreviations used: DOC, deoxycholate; SDS, sodium dodecylsulfate. 


\section{MATERIALS AND METHODS}

Cytosol fraction. Wistar rats weighing 150-250 $\mathrm{g}$ were used, and their pooled livers were homogenized with 4 volumes of $0.25 \mathrm{M}$ sucrose in $10 \mathrm{mM}$ phosphate buffer, $\mathrm{pH}$ 7.0. After centrifugation of the homogenates at $12,000 \times g$ for $15 \mathrm{~min}$, the postmitochondrial supernatant was spun at $105,000 \times g$ for $60 \mathrm{~min}$. The resulting supernatant was used as the cytosol fraction.

pH 6 treatment of the cytosol in the presence of DOC. The cytosol fraction obtained contained about 10 units of catalase per $\mathrm{ml}$ and was diluted with homogenization medium to yield the desired concentrations. One milliliter of diluted cytosol was mixed with $0.1 \mathrm{ml}$ of $10 \%$ DOC, then $0.3 \mathrm{ml}$ of $0.5 \mathrm{M} \mathrm{NaH}_{2} \mathrm{PO}_{4}$ was added. The $\mathrm{pH}$ of the mixture dropped to 6.1 , and precipitates were formed which were separated by centrifugation. These precipitates dissolved readily in $0.2 \mathrm{M}$ Tris buffer, $\mathrm{pH}$ 8.0, after which they were dialyzed against a large volume of a 20 -fold dilution of the buffer.

Purification of the cytosol catalase. Livers from 20 to 30 rats were pooled, and the cytosol fraction treated at $\mathrm{pH} 6$ in the presence of DOC as described above. The precipitates formed were dissolved in $0.2 \mathrm{M}$ Tris, $\mathrm{pH} 8.0$, then dialyzed overnight. A 0.5 volume of acid ethanol solution (44 v/v ethanol in $88 \mathrm{mM}$ acetate buffer, $\mathrm{pH} 4.1$ ) was added to the dialysate, and the resulting precipitates were dissolved in and dialyzed against Tris buffer as before.

After centrifuging off any precipitates, a 0.2 volume of the acid ethanol solution was dropped into the dialysate until it became turbid. About $10 \%$ of the catalase activity was lost with precipitation. A 0.4 volume of the acid ethanol solution was added to the resulting supernatant, then a 0.1 volume of $0.5 \mathrm{M} \mathrm{Na}_{2} \mathrm{SO}_{4}$ was added. This step was very effective for removal of contaminating proteins.

The precipitates first were dissolved in $10 \mathrm{mM}$ phosphate buffer, $\mathrm{pH} 7.0$, then fractionated with ammonium sulfate between 20 and $33 \% \mathrm{w} / \mathrm{v}$. Finally, the preparation was chromatographed on a DEAE-cellulose column as reported previously $(2,3)$.

Enzymatic assay of catalase. Catalase activity was measured spectrophotometrically by following the decrease in hydrogen peroxide at $230 \mathrm{~nm}$ (2).

Polyacrylamide gel electrophoresis. The purified catalase was electrophoresed on $5 \%$ polyacrylamide gel and its activity detected by the method of Woodbury et al. (6). Laemmli's method with a $10 \%$ gel containing sodium dodecylsulfate (SDS) was used to analyze the catalase subunits.

Immunochemical assay of the catalase. Anti-catalase antiserum was prepared against purified peroxisomal catalase as reported previously (2). For the quantitative assay the immunoprecipitates were measured by the method that uses bromsulfophthaleine $(8,9)$. The $\mathrm{A}_{407}$ of the $1 \%$ solution of catalase was assumed to be 1.68 , this value being estimated with the purified peroxisomal catalase (2).

Amino acid composition. Samples were hydrolyzed with $6 \mathrm{~N} \mathrm{HCl}$ for $12 \mathrm{~h}$, then analyzed in a Hitachi 835 amino acid analyzer.

\section{RESULTS}

pH 6 treatment of the cytosol catalase in the presence of DOC. A solution of sodium deoxycholate (DOC) was added to the cytosol obtained from rat liver at a final concentration of $1 \%$, and subsequently the mixture was brought to $\mathrm{pH} 6.1$ by adding $0.5 \mathrm{M} \mathrm{NaH}_{2} \mathrm{PO}_{4}$. The resulting precipitates were centrifuged off at $10,000 \times g$ for $15 \mathrm{~min}$, and the catalase activity in the supernatant was assayed. The catalase in the cytosol was readily precipitated by this $\mathrm{pH} 6$ treatment in the presence of DOC (Fig. 


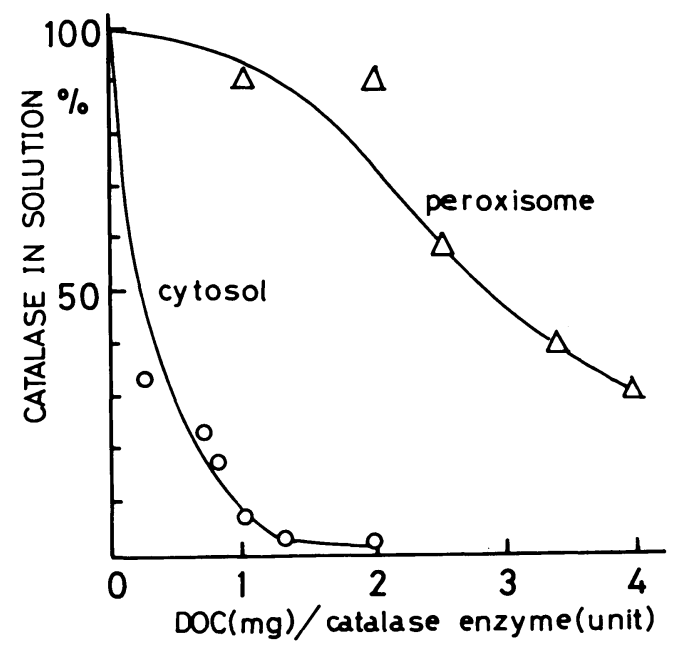

Fig. 1. Precipitation of catalase with DOC at pH 6. (O): catalase in the cytosol fraction, $(\triangle)$ : catalase in the peroxisomal extracts.

1). When the amount of DOC was more than $1 \mathrm{mg}$ per unit of enzyme activity, almost all the catalase was sedimented; but $\mathrm{pH} 6$ treatment of the cytosol without the addition of DOC did not yield any detectable precipitation with catalase activity.

As a comparison, peroxisomes were extracted with various amounts of DOC, and the extracts brought to $\mathrm{pH}$ 6. The results also are shown in Fig. 1; almost no catalase was precipitated when the ratio of DOC $(\mathrm{mg}) /$ enzyme (unit) was unity.

Although as much as $75-90 \%$ of the cytosol catalase was recovered in the precipitates after treatment, only $35-45 \%$ of the $A_{280}$ and about $60 \%$ of the $A_{407}$ were sedimented concomitantly.

Removal of DOC by dialysis. After $\mathrm{pH} 6$ treatment the precipitates formed were

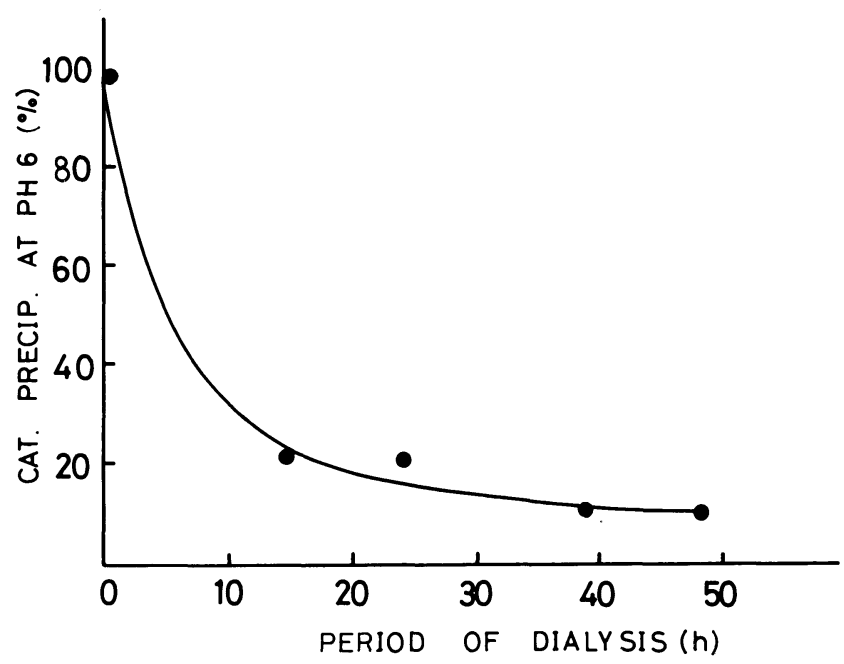

Fig. 2. Removal of DOC by dialysis. For details see the text. 
TABlE 1. Purification OF RAT Liver CytOSOl CATAlases

\begin{tabular}{|c|c|c|c|c|c|}
\hline & Enzyme & $\mathrm{A}_{280}$ & $\mathrm{~A}_{407}$ & $\mathrm{~A}_{407} / \mathrm{A}_{280}$ & * \\
\hline 1. Cytosol & 100 & 100 & 100 & 0.040 & 19.5 \\
\hline 2. DOC, pH 6 treatment & 78 & 41 & 63 & 0.061 & 24.4 \\
\hline 3. Ethanol-acid precipitation & 63 & 21 & 36 & 0.060 & 33.9 \\
\hline 4. Ethanol fractionation, $\mathrm{Na}_{2} \mathrm{SO}_{4}$ & 46 & 1.8 & 12 & 0.269 & 74.8 \\
\hline 5. $\left(\mathrm{NH}_{4}\right)_{2} \mathrm{SO}_{4}$ fractionation & 39 & 1.1 & 10 & 0.363 & 76.0 \\
\hline 6. DEAE-cellulose chromatography & 15 & 0.15 & 3. 7 & 1.00 & 79.4 \\
\hline
\end{tabular}

Enzyme: total catalase activity; $\mathrm{A}_{280}$ : absorbance at $280 \mathrm{~nm}$ (for proteins) $\times$ volume (ml); $\mathrm{A}_{407}$ : absorbance at $407 \mathrm{~nm}$ (for heme) $\times$ volume $(\mathrm{ml})$. These three values in the table are percentages of the original value for the starting cytosol. The column with an asterisk gives values for Enzyme/ A 407.

dissolved in $0.2 \mathrm{M}$ Tris buffer, $\mathrm{pH} 8.0$, then dialyzed against dilute $(0.01 \mathrm{M})$ Tris buffer

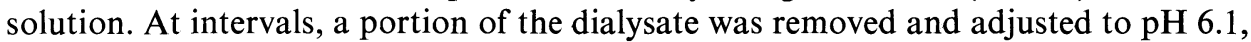
after which the catalase enzyme activity in both the solution and precipitates was measured. Results are shown in Fig. 2. The curve shows the amount of catalase that was precipitated at $\mathrm{pH}$ 6. Probably, the more DOC removed, the less catalase is precipitable at $\mathrm{pH} 6$. After dialysis overnight, nearly $80 \%$ of the catalase was in solution, having survived $\mathrm{pH} 6$ treatment.

Purification of cytosol catalase. Based on the experimental findings described above, we purified the cytosol catalase by a combination of the various techniques

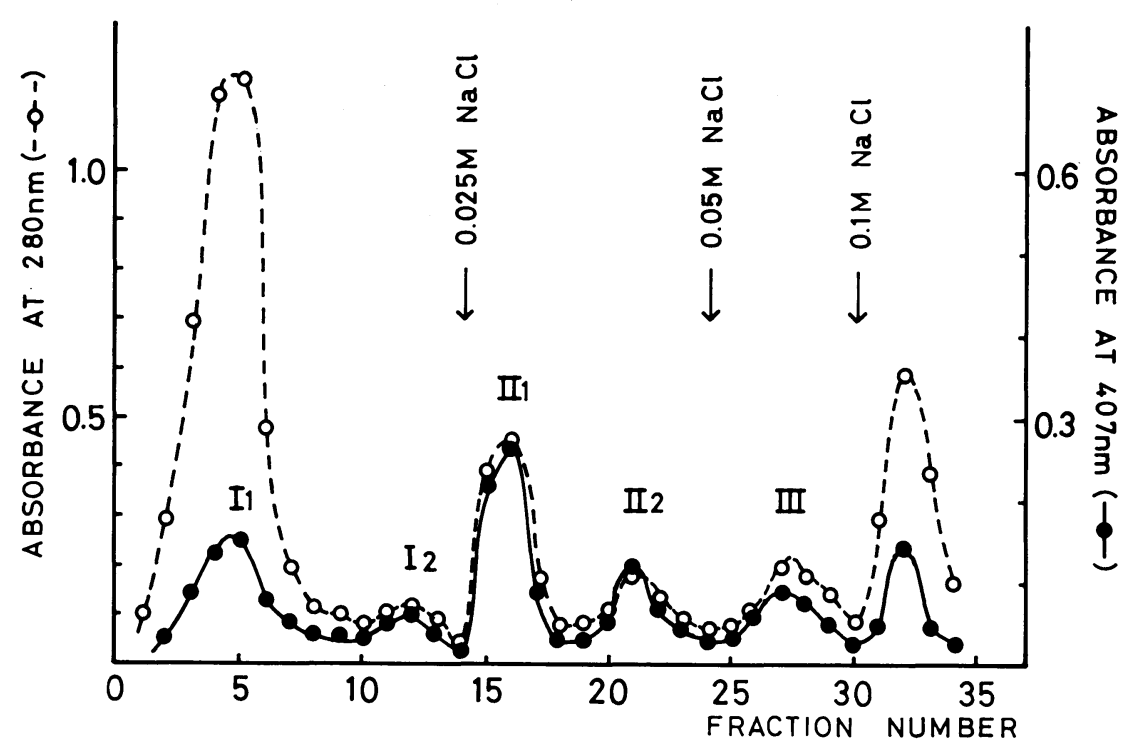

Fig. 3. DEAE-cellulose chromatography of the partially purified cytosol catalase. A DEAEcellulose column was equilibrated with $0.01 \mathrm{M}$ phosphate buffer, $\mathrm{pH} 7.0$, then a partially purified preparation of the cytosol catalase dialyzed against the same buffer was applied to the column. Fractions coming through the column were collected. Then the column was eluted in steps using buffer containing $0.025 \mathrm{M}, 0.05 \mathrm{M}$ and $0.1 \mathrm{M} \mathrm{NaCl}$. Each fraction of the eluate was assayed at $280 \mathrm{~nm}(\bigcirc)$ and at $407 \mathrm{~nm}(\bullet)$. Peaks for $\mathrm{A}_{407}$ contained catalase enzyme activity. 


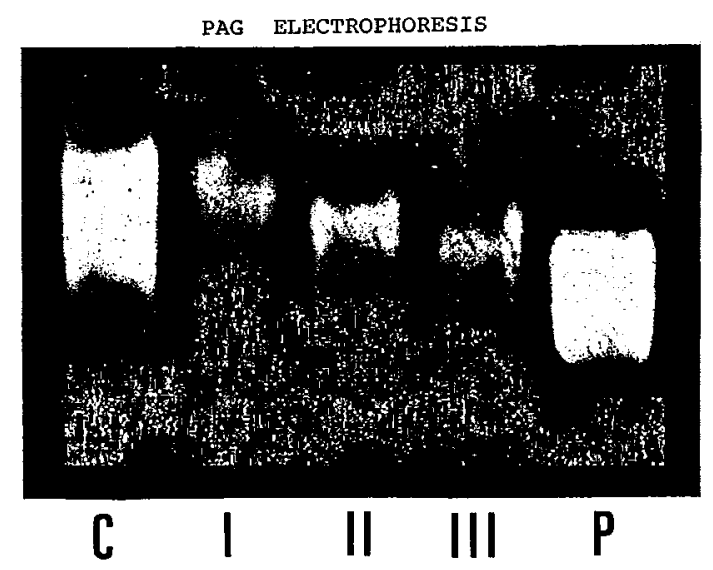

Fig. 4. Polyacrylamide gel electrophoresis of the cytosol and peroxisomal catalases. After electrophoresis the catalase was stained for enzyme activity. C: cytosol fraction, I: cytosol catalase-I, II: cytosol catalase-II, III: cytosol catalase-III and P: purified peroxisomal catalase.

used to purify peroxisomal catalase $(2,5)$. The procedure consisted of $(1)$ coprecipitation with DOC at $\mathrm{pH} 6$, (2) sedimentation by ethanol in acidic buffer, (3) salting out with sodium sulfate in the presence of acid ethanol, (4) fractionation with ammonium sulfate and (5) chromatography on DEAE-cellulose. Details are described in MATERIALS AND METHODS.

Recovery of enzyme activity, $\mathrm{A}_{280}$ (for proteins) and $\mathrm{A}_{403}$ (for heme) after each step of purification is shown in Table 1 . The ratios of $A_{407} / A_{280}$, one criterion for the purity of catalase, and the specific enzyme activities (units/ $A_{407}$ ) are also shown in the table. These values for the final preparation coincide with those of the purified peroxisomal catalase (2).

Heterogeneity of the cytosol catalase. Chromatography of the cytosol catalase on

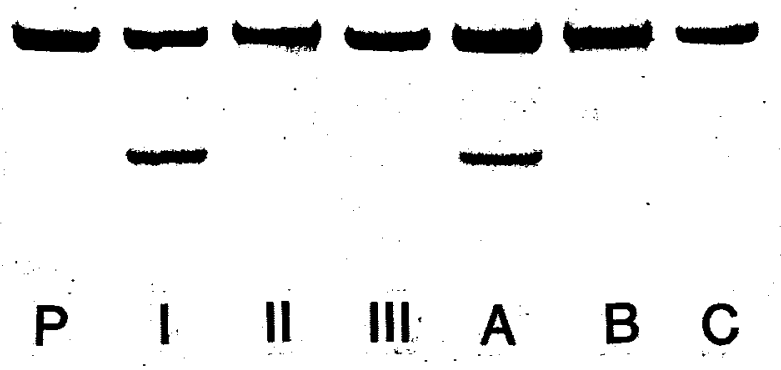

Fig. 5. SDS polyacrylamide gel electrophoresis of the cytosol and peroxisomal catalases. The gel was stained with Coomassie brilliant blue. Specimens I to III and $\mathrm{P}$ are the respective purified preparations of the cytosol catalases -I, II- and -III and of the peroxisomal catalase. A: I+P, B: II+III and C: a mixture of all four preparations. 
DEAE-cellulose revealed its heterogeneity (Fig. 3). About $30 \%$ did not stick to a column equilibrated with $0.01 \mathrm{M}$ phosphate buffer, $\mathrm{pH} 7.0$ (designated $\mathrm{I}-1,2$ ), and approximately $50 \%$ of the catalase was eluted with $0.025 \mathrm{M} \mathrm{NaCl}$ in the buffer (designated II-1, 2). Another portion, estimated to be nearly $20 \%$ of the total, was recovered in the eluate with $0.05 \mathrm{M} \mathrm{NaCl}$ (designated III). These subfractions of the cytosol catalase have been purified separately by repeated chromatography.

Characterization of the heterogeneous cytosol catalase. The difference in electrophoretic mobility among the heterogeneous cytosol catalases is shown in Fig. 4. The polyacrylamide gel was stained for catalase enzyme activity. As expected from the elution pattern shown in Fig. 3, catalase-I is more alkaline, -II is intermediate and -III is more acidic. The peroxisomal catalase moved faster than these cytosol catalases.

On SDS polyacrylamide gel electrophoresis the three cytosol catalases gave bands identical to that of the peroxisomal catalase. The identical bands represent the subunit, four molecules of which assemble to form one molecule of catalase (see Fig. 5). No molecule larger than this subunit was detected, but the preparation of catalase-I gave a few protein bands in the area of a smaller size molecule. These latter bands may include degradation products formed during storage.

Catalases-I through -III were immunologically identical with each other and with the peroxisomal enzyme on agar diffusion (Fig. 6). The precipitin lines were completely fused, and a second weaker line usually seen with the crude cytosol fraction did not appear in any of the preparations of catalase-I, -II or -III.

The amino acid compositions of the two purified cytosol catalases (II-1 and III) are given in Table 2 and that of the peroxisomal catalase reported previously (10) is

TABLE 2. AMINO ACID COMPOSITION OF THE CYTOSOL AND PEROXISOMAL CATALASES

\begin{tabular}{|c|c|c|c|}
\hline \multirow{2}{*}{ Amino acid } & \multicolumn{2}{|c|}{ Cytosol catalase } & \multirow{2}{*}{$\begin{array}{l}\text { Peroxisomal } \\
\text { catalase }\end{array}$} \\
\hline & II & III & \\
\hline Aspartic acid & 13. 21 & 13.54 & 13.05 \\
\hline Threonine & 4. 96 & 4.93 & 4. 90 \\
\hline Serine & 4. 56 & 4. 48 & 4. 76 \\
\hline Glutamic acid & 10.54 & 10.63 & 10.53 \\
\hline Glycine & 6.87 & 7.08 & 6.78 \\
\hline Alanine & 8.09 & 7.87 & 7.70 \\
\hline Valine & 6.38 & 6.25 & 6.73 \\
\hline Cysteine & 0.46 & 0.43 & 0.53 \\
\hline Methionine & 2.03 & 1.99 & 2.36 \\
\hline Isoleucine & 3. 71 & 3.67 & 3. 79 \\
\hline Leucine & 6.49 & 6.21 & 6.16 \\
\hline Tyrosine & 3.89 & 3.94 & 3.65 \\
\hline Phenylalanine & 6.05 & 6.34 & 5.85 \\
\hline Lysine & 6.12 & 5.85 & 5.95 \\
\hline Histidine & 3.64 & 3.66 & 3.69 \\
\hline Arginine & 5.91 & 6.04 & 5.66 \\
\hline Proline & 7.25 & 7.27 & 8.06 \\
\hline
\end{tabular}

Values are expressed in mole \%, excluding tryptophan. These data are averages of two separate preparations. 


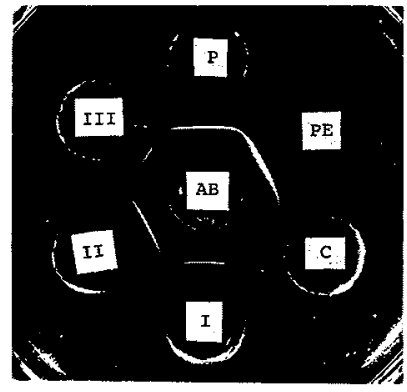

Fig. 6. Agar diffusion of the cytosol and peroxisomal catalases. AB: antiserum against purified peroxisomal catalase, $\mathrm{PE}$ : DOC-extracts of peroxisomes, $\mathrm{P}$ : purified peroxisomal catalase, $\mathrm{C}$ : cytosol fraction and I to III: cytosol catalase-I, -II and -III.

shown for comparison. Catalase-II and -III have similar constituents, whereas the peroxisomal catalase seems to have slight differences in the contents of a few of its amino acids in comparison with the amino acids of the two cytosol catalases.

\section{DISCUSSION}

Because the purification of rat liver catalase has been performed only with the peroxisomal enzyme, our report is the first to describe purified cytosol catalase. The many kinds of proteins present in the supernatant fraction of liver homogenates have prevented us from isolating the catalase in the same compartment of the cell. Coprecipitation of catalase with DOC overcomes this difficulty. The preparations of purified cytosol catalase obtained in our study showed a value of $C a .1 .0$ for $\mathrm{A}_{407} / \mathrm{A}_{280}$, which value is compatible with that for the purified peroxisomal catalase $(2,5)$. In terms of enzyme activity units per $\mathrm{A}_{407}$, values were as high as that of the peroxisomal catalase. Gel electrophoresis in the presence of SDS gave a single band corresponding to the subunit of catalase. Therefore, except for catalase-I, the cytosol catalases are considered to have been extensively purified.

Peroxisomal catalase routinely is extracted from the organelle with DOC or Triton. To remove DOC, the extract is brought to $\mathrm{pH} \mathrm{6,} \mathrm{at} \mathrm{which} \mathrm{pH}$ DOC is sedimented and the catalase remains in solution. In the experiment done to find the difference between the peroxisomal and cytosol catalase, we found that the cytosol catalase precipitates with DOC at $\mathrm{pH}$ 6. This apparent difference between the two catalases was investigated, and the following evidence obtained: Peroxisomal catalase is itself precipitable with DOC at $\mathrm{pH} 6$, but not in the presence of membrane fractions of the organelle, whereas cytosol catalase always is precipitated with DOC even when membrane fractions of the peroxisomes are added. Details of this investigation will be reported elsewhere.

An earlier report by Higashi (3) demonstrated that peroxisomal catalase is trapped by DEAE-cellulose more tightly than is cytosol catalase. Peroxisomal catalase was eluted from a column of DEAE-cellulose equilibrated with $0.01 \mathrm{M}$ phosphate buffer, $\mathrm{pH} 7.0$, by $0.1 \mathrm{M}$ sodium chloride. In the study we report here, some of the cytosol catalase came through the column under the same condition but most of the remainder was eluted by $\mathrm{NaCl}$ at less than $0.1 \mathrm{M}$. The lower acidity of the cytosol catalase in comparison to that of the peroxisomal catalase, which was expected from its chro- 
matographic behaviour, has been shown previously by electrophoresis of preparations purified by immunoaffinity chromatography (4).

Heterogeneous components of the cytosol catalase showed differences in electrophoretic mobility, all moved slower than the peroxisomal catalase (Fig. 4). According to the findings of Mainferme and Wattiaux (10), the peroxisomal catalase has, itself, the same electrophoretic mobility as the cytosol catalase, the more anodic molecule being a modified product when proteolytic cleavage by a lysosomal enzyme takes place during isolation procedures. This modified peroxisomal catalase principally has been observed in the purified catalase from the conventional mitochondrial fraction. Its molecular weight is 2,500 daltons less than that of the native enzyme. Based on their experimental results, Robbi and Lazarow (11) concluded that a decrease in molecular weight of 4,000 daltons takes place in the peroxisomal catalase during purification. A similar modified molecule of 2,000 daltons less has been described by Crane et al. (12) for mouse liver catalase. In our experiments, no difference in size has been found so far for the peroxisomal catalase immunoprecipitated from crude extracts in the presence (4) or absence (Fig. 5) of leupeptin, the chemically purified peroxisomal catalase and the cytosol catalase, but a detailed reinvestigation is being made.

The amino acid composition of the cytosol catalase reported here differs slightly from that of the peroxisomal catalase previously studied (13). There may be an exchange of a few amino acids between the two catalases. If so, the candiates are assumed to be alanine for proline, leucine for valine, tyrosine for serine and arginine for methionine. An exchange of one or two amino acid(s) may take place between the cytosol catalase II and III, i.e. leucine for phenylalanine and alanine for aspartic acid or glycine. These possibilities are worth investigating.

Based on the evidence obtained so far we now consider that the cytosol catalase is not the same as the peroxisomal catalase. Neither an extra peptide nor sugar components have been detected in the molecule of the two forms of rat liver catalase. They are probably synthesized under the direction of different messenger RNAs. The peroxisomal catalase may possess a particular structure that recognizes a specific site on the membrane of the peroxisome, which is missing in the cytosol catalase. It should not be difficult to find this structure in fragments of the molecule prepared by various treatments. This is the next subject in our study.

Acknowledgements. We are grateful to Professor T. Sato and Mr. T. Kadofuku of Showa University for their analyses of the amino acid compositions.

\section{REFERENCES}

1. Ludewig, S. and A. Chanutin. Distribution of enzymes in the livers of control and X-irradiated rats. Arch. Biochem. 29, 441-445, 1950

2. Higashi, T. and T. Peters, JR. Studies on rat liver catalase. I. Combined immunochemical and enzymatic determination of catalase in liver cell fractions. J. Biol. Chem. 238, 3945-3951, 1963

3. Higashi, T. and Y. Shibata. Studies on rat liver catalase. IV. Heterogeneity of mitochondrial and supernatant catalase. J. Biochem. 58, 530-537, 1965

4. Sugita, Y., T. Tobe, T. Sakamoto and T. Higashi. Immature precursor catalase in subcellular fractions of rat liver. J. Biochem. 92, 509-515, 1982

5. Price, V.E. and R.E. GreEnfield. Liver catalase. II. Catalase fractions from normal and tumorbearing rats. J. Biol. Chem. 209, 363-376, 1954 
6. Woodbury, W., A.K. Spencer and M.A. Stahmann. An improved procedure using ferricyanide for detecting catalase isozymes. Anal. Biochem. 44, 301-305, 1971

7. LAemmLi, U.K. Cleavage of structural proteins during the assembly of the head of bacteriophage $\mathrm{T}_{4}$. Nature 227, 680-685, 1970

8. Shibata, Y. and T. Higashi. Studies on the precipitin reaction of rat catalase. Seibutsu-Butsurikagaku 11, 259-266, 1966 (in Japanese)

9. Glick, D., A.R. Good, L.J. Greenberg, J.J. Eddy and N.K. DAY. Measurement of precipitin reactions in the millimicrogram protein nitrogen range. Science 128, 1625-1626, 1958

10. Mainferme, F. and R. Wattiaux. Effect of lysosomes on rat-liver catalase. Europ. J. Biochem. 127, 343-346, 1982

11. Roвbi, M. and P.B. Lazarow. Synthesis of catalase in two cell-free protein-synthesizing systems and in rat liver. Proc. Natl. Acad. Sci. U.S.A. 75, 4344-4348, 1978

12. Crane, D., R. Holmes and C. Masters. Proteolytic modification of mouse liver catalase. Biochem. Biophys. Res. Commun. 104, 1567-1572, 1982

13. Higashi, T. and Y. Shibata. Studies on rat liver catalase. III. Amino acid composition of rat liver catalase. J. Biochem. 56, 361-363, 1964 